03

\title{
Применение метода продолженных граничных условий к решению задачи дифракции волн на рассеивателях сложной геометрии, расположенных в однородной и неоднородной средах
}

\author{
(C) Д.В. Крысанов ${ }^{1}$, А.Г. Кюркчан ${ }^{1,2,3}$, С.А. Маненков ${ }^{1}$ \\ ${ }^{1}$ Московский технический университет связи и инорорматики, \\ 111024 Москва, Россия \\ ${ }^{2}$ Фрязинский филиал Института радиотехники и электроники им. В.А. Котельникова РАН, \\ 141190 Фрязино, Московская обл., Россия \\ ${ }^{3}$ Центральный научно-исследовательский институт связи, \\ 111141 Москва, Россия \\ e-mail: dimok1993@mail.ru
}

Поступила в редакцию 04.12.2019 г.

В окончательной редакции 04.12.2019 г.

Принята к публикации 23.12.2019 г.

На основе метода продолженных граничных условий предложена методика, позволяющая моделировать характеристики рассеяния для тел произвольной геометрии. В работе рассмотрена двумерная задача дифракции плоской волны на диэлектрических телах со сложной геометрией сечения, в частности на фракталоподобных телах. Проведено сравнение численных алгоритмов решения задачи дифракции на основе систем интегральных уравнений 1-го и 2-го рода. Приведено обобщение метода на задачу дифракции на цилиндрическом теле, расположенном в однородном магнитодиэлектрическом полупространстве. Корректность метода подтверждена при помощи проверки выполнения оптической теоремы для различных тел и путем сравнения с результатами расчетов, полученных модифицированным методом дискретных источников.

Ключевые слова: дифракция волн на телах сложной геометрии, метод продолженных граничных условий, дифракция на телах, расположенных в плоскослоистой среде.

DOI: $10.21883 /$ OS.2020.04.49199.327-19

\section{Введение}

Задача дифракции волн на диэлектрическом теле сложной геометрии является весьма актуальной и остается сравнительно слабо исследованной ввиду сложности ее решения. Результаты моделирования характеристик рассеяния волн диэлектрическими телами представляют большой интерес в таких, например, областях, как оптика неоднородных сред, лазерная дефектоскопия, проектирование поглощающих покрытий и др. [1-3]. Несмотря на то, что на данный момент разработан ряд аналитических и численных методов решения этих задач (наиболее распространенными из которых являются метод Т-матриц [4] и метод дискретных источников [5]), потребности в моделировании дифракционных процессов возрастают довольно быстро, в связи с чем вопрос разработки более универсальных методов решения задач дифракции все еще остается актуальным. Широкая популярность метода Т-матриц объясняется во многом тем, что с использованием этого метода можно сравнительно легко выполнять такую важную, например, в астрофизике процедуру, как усреднение характеристик рассеяния тела по углам его ориентации относительно падающей плоской волны. Однако традиционный (классический) вариант метода Т-матриц [4], как и некоторые его недавно разработанные модифицированные вариан- ты $[5,6]$, применимы к решению задач дифракции лишь на рассеивателях с аналитической границей.

В работах [7,8] было предложено обобщение метода Т-матриц на основе метода продолженных граничных условий (МПГУ) для решения задачи дифракции с условием Дирихле на границе в двумерном и трехмерном случаях. Двумерный случай был также рассмотрен для импедансного краевого условия [9]. Идея МПГУ заключается в переносе граничного условия с поверхности $S$ рассеивателя на некоторую вспомогательную поверхность $S_{\delta}$, которая располагается вне рассеивателя на некотором достаточно малом расстоянии $\delta$ от его границы. К главным достоинствам МПГУ можно отнести отсутствие ограничений на геометрию рассеивателя (в том числе он применим и для рассеивателей, имеющих изломы границы, и для тонких экранов). Кроме того, МПГУ предлагает единый подход к решению краевых задач, не зависящий от их типа, размерности, геометрии поверхности рассеивателя и характера рассеиваемого поля. Отметим также, что в рамках МПГУ задача дифракции может быть сведена к решению системы интегральных уравнений 1- или 2-рода, что невозможно также просто осуществить, например, при решении задачи методом поверхностных интегральных уравнений.

В настоящей работе предлагается обобщение описанной выше методики для решения двумерной задачи ди- 


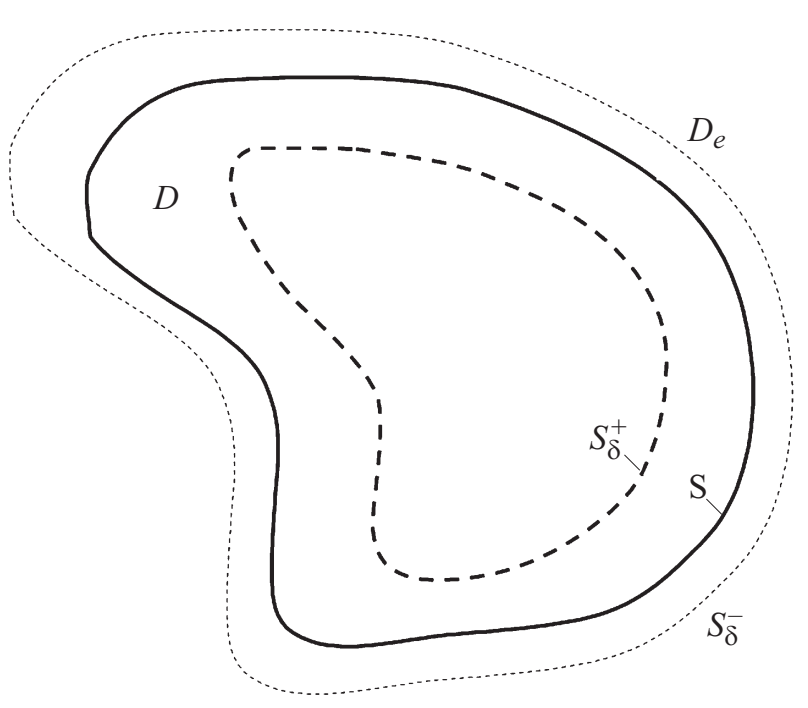

Рис. 1. Геометрия задачи дифракции на теле, расположенном в однородной среде.

фракции электромагнитных волн на диэлектрическом теле. Рассмотрены примеры моделирования характеристик рассеяния волн телами с поперечным сечением сложной геометрии и фракталоподобными телами. Приведены формулы и результаты расчетов диаграммы рассеяния тел сложной геометрии, расположенных в однородном диэлектрическом полупространстве.

\section{1. Вывод основных соотношений}

Пусть на бесконечно длинный магнитодиэлектрический цилиндр с образующей, параллельной оси $O Z$, и с направляющей $S$ падает первичное электромагнитное поле $\mathbf{E}^{0}, \mathbf{H}^{0}$. Геометрия задачи изображена на рис. 1. Рассмотрим случай $E$-поляризации, когда вектор напряженности электрического поля $\mathbf{E}$ имеет только одну составляющую $E_{z}$ (ниже обозначаемую буквой $U_{-}$ или $U_{+}$), параллельную образующей цилиндрического тела. Тогда на границе рассеивателя будут иметь место следующие условия сопряжения:

$$
\left.U_{+}\right|_{S}=\left.U_{-}\right|_{S},\left.\quad \frac{\partial U_{+}}{\partial n}\right|_{S}=\left.\kappa \frac{\partial U_{-}}{\partial n}\right|_{S},
$$

где $U_{+}$- поле внутри цилиндра, $U_{-}=U^{0}+U^{1}-$ полное поле вне тела, причем $U^{0}$ - падающее, а $U^{1}-$ рассеянное (вторичное) поле, $\frac{\partial}{\partial n}-$ дифференцирование по направлению внешней к $S$ нормали, $\boldsymbol{\kappa}=\mu_{i} / \mu_{e}$, $\mu_{i}$ и $\mu_{e}-$ относительные магнитные проницаемости сред внутри и вне тела соответственно. Внешняя среда $\left(D_{e}=\mathscr{R}^{2} \backslash \bar{D}, \bar{D}=D \cup S, D-\right.$ область, ограниченная кривой $S$ ) и среда внутри цилиндра полагаются однородными, линейными и изотропными. На бесконечности предполагаются выполненными стандартные условия излучения для рассеянного поля.
Воспользуемся следующими представлениями для решения уравнения Гельмгольца в областях $D$ и $D_{e}$ соответственно [5]:

$$
\begin{aligned}
U_{-}(\mathbf{r}) & =U^{0}(\mathbf{r}) \\
& +\int_{S}\left\{\frac{\partial U_{-}\left(\mathbf{r}^{\prime}\right)}{\partial n^{\prime}} G_{-}\left(\mathbf{r}, \mathbf{r}^{\prime}\right)-U_{-}\left(\mathbf{r}^{\prime}\right) \frac{\partial G_{-}\left(\mathbf{r} ; \mathbf{r}^{\prime}\right)}{\partial n^{\prime}}\right\} d s^{\prime}, \\
U_{+}(\mathbf{r}) & =-\int_{S}\left\{\frac{\partial U_{+}\left(\mathbf{r}^{\prime}\right)}{\partial n^{\prime}} G_{+}\left(\mathbf{r}, \mathbf{r}^{\prime}\right)-U_{+}\left(\mathbf{r}^{\prime}\right) \frac{\partial G_{+}\left(\mathbf{r} ; \mathbf{r}^{\prime}\right)}{\partial n^{\prime}}\right\} d s^{\prime},
\end{aligned}
$$

в которых $G_{ \pm}\left(\mathbf{r} ; \mathbf{r}^{\prime}\right)=\frac{1}{4 i} H_{0}^{(2)}\left(k_{ \pm}\left|\mathbf{r}-\mathbf{r}^{\prime}\right|\right)-$ это фундаментальные решения скалярного уравнения Гельмгольца в $\mathscr{R}^{2}$ с материальными параметрами сред $D_{e}$ и $D$ соответственно, причем $k_{+}$и $k_{-}-$волновые числа среды внутри и вне рассеивателя. Потребовав в соответствии с МПГУ выполнения условий (1) на контуре $S_{\delta}^{-}$, расположенном в $\mathscr{R}^{2} \backslash \bar{D}$, и на контуре $S_{\delta}^{+}$, расположенном в области $D$ (рис. 1 ), с использованием соотношений (2), получим следующие системы интегральных уравнений (СИУ) Фредгольма 1-го или 2-го рода соответственно:

$$
\begin{aligned}
& \int_{S}\left\{\frac{\partial U\left(\mathbf{r}^{\prime}\right)}{\partial n^{\prime}}\left(G_{-}\left(\mathbf{r}_{-} ; \mathbf{r}^{\prime}\right)+\kappa G_{+}\left(\mathbf{r}_{+} ; \mathbf{r}^{\prime}\right)\right)\right. \\
& \left.-U\left(\mathbf{r}^{\prime}\right)\left(\frac{\partial G_{-}\left(\mathbf{r}_{-} ; \mathbf{r}^{\prime}\right)}{\partial n^{\prime}}+\frac{\partial G_{+}\left(\mathbf{r}_{+} ; \mathbf{r}^{\prime}\right)}{\partial n^{\prime}}\right)\right\} d s^{\prime}=-U^{0}\left(\mathbf{r}_{-}\right), \\
& \int_{S}\left\{\frac{\partial U\left(\mathbf{r}^{\prime}\right)}{\partial n^{\prime}}\left(\frac{\partial G_{-}\left(\mathbf{r}_{-} ; \mathbf{r}^{\prime}\right)}{\partial n}+\frac{\partial G_{+}\left(\mathbf{r}_{+} ; \mathbf{r}^{\prime}\right)}{\partial n}\right)\right. \\
& -U\left(\mathbf{r}^{\prime}\right)\left(\frac{\partial^{2} G_{-}\left(\mathbf{r}_{-} ; \mathbf{r}^{\prime}\right)}{\partial n \partial n^{\prime}}+\frac{1}{\kappa} \frac{\partial^{2} G_{+}\left(\mathbf{r}_{+} ; \mathbf{r}^{\prime}\right)}{\partial n \partial n^{\prime}}\right) d s^{\prime}= \\
& -\frac{\partial U^{0}\left(\mathbf{r}_{-}\right)}{\partial n} \\
& U(\mathbf{r})=\frac{1}{2} U^{0}\left(\mathbf{r}_{-}\right) \\
& +\frac{1}{2} \int_{S}\left\{\frac{\partial U\left(\mathbf{r}^{\prime}\right)}{\partial n^{\prime}}\left(G_{-}\left(\mathbf{r}_{-} ; \mathbf{r}^{\prime}\right)-\kappa G_{+}\left(\mathbf{r}_{+} ; \mathbf{r}^{\prime}\right)\right)\right. \\
& \left.-U\left(\mathbf{r}^{\prime}\right)\left(\frac{\partial G_{-}\left(\mathbf{r}_{-} ; \mathbf{r}^{\prime}\right)}{\partial n^{\prime}}-\frac{\partial G_{+}\left(\mathbf{r}_{+} ; \mathbf{r}^{\prime}\right)}{\partial n^{\prime}}\right)\right\} d s^{\prime}, \\
& \frac{\partial U(\mathbf{r})}{\partial n}=\frac{1}{1+\kappa} \frac{\partial U^{0}\left(\mathbf{r}_{-}\right)}{\partial n}+\frac{1}{1+\kappa} \\
& \times \int_{S}\left\{\frac{\partial U\left(\mathbf{r}^{\prime}\right)}{\partial n^{\prime}}\left(\frac{\partial G_{-}\left(\mathbf{r}_{-} ; \mathbf{r}^{\prime}\right)}{\partial n}-\kappa \frac{\partial G_{+}\left(\mathbf{r}_{+} ; \mathbf{r}^{\prime}\right)}{\partial-n}\right)\right. \\
& \left.-U\left(\mathbf{r}^{\prime}\right)\left(\frac{\partial^{2} G_{-}\left(\mathbf{r}_{-} ; \mathbf{r}^{\prime}\right)}{\partial n \partial n^{\prime}}-\frac{\partial^{2} G_{+}\left(\mathbf{r}_{+} ; \mathbf{r}^{\prime}\right)}{\partial n \partial n^{\prime}}\right)\right\} d s^{\prime},
\end{aligned}
$$

где точки наблюдения $M\left(\mathbf{r}_{ \pm}\right)$принадлежат контурам $S_{\delta}^{+}$, а точка $M(\mathbf{r}) \in S$ и обозначено $U=U_{-}$. Отметим, что чаще всего в качестве $S_{\delta}^{ \pm}[5,10]$ выбирают контуры, 
отстоящие от $S$ на некоторое достаточно малое расстояние $\delta$, т. е. рассматривают эквидистантные контуры. Пусть уравнение границы $S$ задано в параметрическом виде

$$
\left\{\begin{array}{l}
x=x^{\prime}(t), \\
y=y^{\prime}(t), t \in\left[0, t_{\max }\right] .
\end{array}\right.
$$

Тогда уравнения смещенных контуров $S^{\mp}$ записываются следующим образом:

$$
\left\{\begin{array}{l}
x=x^{\prime}(t) \pm n_{x}(t) \delta \\
y=y^{\prime}(t) \pm n_{y}(t) \delta
\end{array}\right.
$$

где $n_{x}$ и $n_{y}-$ координаты нормали к границе тела $S$. Для решения систем (3) и (4) используем метод КрыловаБоголюбова. Для этого запишем системы уравнений (3) и (4) в виде

$$
\begin{gathered}
\int_{0}^{t_{\max }} K_{11}\left(t, t^{\prime}\right) I_{1}\left(t^{\prime}\right)+K_{12}\left(t, t^{\prime}\right) I_{2}\left(t^{\prime}\right) d t^{\prime}=b_{1}(t), \\
\int_{0}^{t_{\max }} K_{21}\left(t, t^{\prime}\right) I_{1}\left(t^{\prime}\right)+K_{22}\left(t, t^{\prime}\right) I_{2}\left(t^{\prime}\right) d t^{\prime}=b_{2}(t), \\
I_{1}(t)+\int_{0}^{t_{\max }} \tilde{K}_{11}\left(t, t^{\prime}\right) I_{1}\left(t^{\prime}\right)+\tilde{K}_{12}\left(t, t^{\prime}\right) I_{2}\left(t^{\prime}\right) d t^{\prime}=\tilde{b}_{1}(t), \\
I_{2}(t)+\int_{0}^{t_{\max }} \tilde{K}_{21}\left(t, t^{\prime}\right) I_{1}\left(t^{\prime}\right)+\tilde{K}_{22}\left(t, t^{\prime}\right) I_{2}\left(t^{\prime}\right) d t^{\prime}=\tilde{b}_{2}(t),
\end{gathered}
$$

в которых

$$
\begin{gathered}
I_{1}\left(t^{\prime}\right)=U\left(\mathbf{r}^{\prime}\left(t^{\prime}\right)\right), I_{2}\left(t^{\prime}\right)=\frac{\partial U\left(\mathbf{r}^{\prime}\left(t^{\prime}\right)\right)}{\partial n^{\prime}} \\
\xi\left(t^{\prime}\right)=\sqrt{\dot{x}^{\prime}\left(t^{\prime}\right)^{2}+\dot{y}^{\prime}\left(t^{\prime}\right)^{2}} \\
K_{11}=-\left(\frac{\partial G_{-}\left(\mathbf{r}_{-} ; \mathbf{r}^{\prime}\right)}{\partial n^{\prime}}+\frac{\partial G_{+}\left(\mathbf{r}_{+} ; \mathbf{r}^{\prime}\right)}{\partial n^{\prime}}\right) \xi\left(t^{\prime}\right), \\
K_{12}=\left(G_{-}\left(\mathbf{r}_{-} ; \mathbf{r}^{\prime}\right)+\kappa G_{+}\left(\mathbf{r}_{+} ; \mathbf{r}^{\prime}\right)\right) \xi\left(t^{\prime}\right) \\
K_{21}=-\left(\frac{\partial^{2} G_{-}\left(\mathbf{r}_{-} ; \mathbf{r}^{\prime}\right)}{\partial n \partial n^{\prime}}+\frac{1}{\kappa} \frac{\partial^{2} G_{+}\left(\mathbf{r}_{+} ; \mathbf{r}^{\prime}\right)}{\partial n \partial n^{\prime}}\right) \xi\left(t^{\prime}\right), \\
K_{22}=\left(\frac{\partial G_{-}\left(\mathbf{r}_{-} ; \mathbf{r}^{\prime}\right)}{\partial n}+\frac{\partial G_{+}\left(\mathbf{r}_{+} ; \mathbf{r}^{\prime}\right)}{\partial n}\right) \xi\left(t^{\prime}\right) \\
b_{1}(t)=-U^{0}(\mathbf{r}(t)), b_{2}(t)=-\frac{\partial U^{0}\left(\mathbf{r}_{-}(t)\right)}{\partial n} \\
\tilde{K}_{11}=-\frac{1}{2}\left(\frac{\partial G_{-}\left(\mathbf{r}_{-} ; \mathbf{r}^{\prime}\right)}{\partial n^{\prime}}-\frac{\partial G_{+}\left(\mathbf{r}_{+} ; \mathbf{r}^{\prime}\right)}{\partial n^{\prime}}\right) \xi\left(t^{\prime}\right) \\
\tilde{K}_{12}=\frac{1}{2}\left(G_{-}\left(\mathbf{r}_{-} ; \mathbf{r}^{\prime}\right)+\kappa G_{+}\left(\mathbf{r}_{+} ; \mathbf{r}^{\prime}\right)\right) \xi\left(t^{\prime}\right) \\
\tilde{K}_{21}=\frac{1}{1+\kappa}\left(\frac{\partial^{2} G_{-}\left(\mathbf{r}_{-} ; \mathbf{r}^{\prime}\right)}{\partial n \partial n^{\prime}}-\frac{\partial^{2} G_{+}\left(\mathbf{r}_{+} ; \mathbf{r}^{\prime}\right)}{\partial n \partial n^{\prime}}\right) \xi\left(t^{\prime}\right),
\end{gathered}
$$

$$
\begin{gathered}
\tilde{K}_{22}=\frac{1}{1+\kappa}\left(\frac{\partial G_{-}\left(\mathbf{r}_{-} ; \mathbf{r}^{\prime}\right)}{\partial n}-\kappa \frac{\partial G_{+}\left(\mathbf{r}_{+} ; \mathbf{r}^{\prime}\right)}{\partial n}\right) \xi\left(t^{\prime}\right), \\
\tilde{b}_{1}(t)=-\frac{1}{2} U^{0}\left(\mathbf{r}_{-}(t)\right), \tilde{b}_{2}(t)=\frac{1}{1+\kappa} \frac{\partial U^{0}\left(\mathbf{r}_{-}(t)\right)}{\partial n} .
\end{gathered}
$$

Точка в (9) означает производную по $t$. Представим далее неизвестные функции $I_{1,2}(t)^{\prime}$ в виде сумм

$$
I_{q} \cong \sum_{n=1}^{N} c_{n}^{q} \Phi_{n}\left(t^{\prime}\right), \quad q=1,2,
$$

где $\Phi_{n}\left(t^{\prime}\right)$ - импульсные функции:

$$
\Phi_{n}(t)^{\prime}= \begin{cases}1, & t \in\left[t_{n}-\Delta / 2, t_{n}+\Delta / 2\right], \\ 0, & t \notin\left[t_{n}-\Delta / 2, t_{n}+\Delta / 2\right] .\end{cases}
$$

Здесь $t_{n}=\frac{t_{\max }}{N}\left(n-\frac{1}{2}\right), n=\overline{1, N}, \Delta=\frac{t_{\max }}{N}-$ шаг сетки, $N$ - число базисных функций. Далее, подставив (11) в системы интегральных уравнений (7) и (8) и приравняв левую и правую части в точках коллокации с координатами $\left(x\left(t_{n}\right), y\left(t_{n}\right)\right)$, выбранных на кривых, получим следующие системы алгебраических уравнений относительно величин $c_{n}^{q}$ :

$$
\begin{gathered}
\sum_{n=1}^{N}\left(K_{m n}^{11} c_{n}^{1}+K_{m n}^{12} c_{n}^{2}\right)=b_{m}^{1}, \\
\sum_{n=1}^{N}\left(K_{m n}^{21} c_{n}^{1}+K_{m n}^{22} c_{n}^{2}\right)=b_{m}^{2}, \quad m=\overline{1, N},
\end{gathered}
$$

или

$$
\begin{gathered}
c_{m}^{1}+\sum_{n=1}^{N}\left(\tilde{K}_{m n}^{11} c_{n}^{1}+\tilde{K}_{m n}^{12} c_{n}^{2}\right)=\tilde{b}_{m}^{1}, \\
c_{m}^{2}+\sum_{n=1}^{N}\left(\tilde{K}_{m n}^{21} c_{n}^{1}+\tilde{K}_{m n}^{22} c_{n}^{2}\right)=\tilde{b}_{m}^{2}, \quad m=\overline{1, N},
\end{gathered}
$$

где матричные элементы и правые части вычисляются по формулам

$$
\begin{gathered}
K_{m n}^{p q}=\int_{t_{n}-\Delta / 2}^{t_{n}+\Delta / 2} K_{p q}\left(t_{m}, t\right) d t, \quad b_{m}^{p}=b_{p}\left(t_{m}\right), \\
\tilde{K}_{m n}^{p q}=\int_{t_{n}-\Delta / 2}^{t_{n}+\Delta / 2} \tilde{K}_{p q}\left(t_{m}, t\right) d t, \tilde{b}_{m}^{p}=\tilde{b}_{p}\left(t_{m}\right), p, q=1,2 .
\end{gathered}
$$

Переходя к асимптотике рассеянного волнового поля при $\mathbf{r} \rightarrow \infty$ с учетом формул (2), (5), (11) и (12) получим следующее выражение для диаграммы рассеяния:

$$
\begin{aligned}
g(\varphi) & =\frac{i \Delta}{4} \sum_{n=1}^{N}\left(c_{n}^{2}-c_{n}^{1} i k_{-}\left(n_{x}^{\prime}\right)\left(t_{n}\right) \cos \varphi\right. \\
& \left.+n_{y}^{\prime}\left(t_{n} \sin \varphi\right)\right) \xi\left(t_{n}\right) e^{i k_{-}\left(x^{\prime}\left(t_{n}\right) \cos \varphi+y^{\prime}\left(t_{n} \sin \varphi\right)\right)} .
\end{aligned}
$$




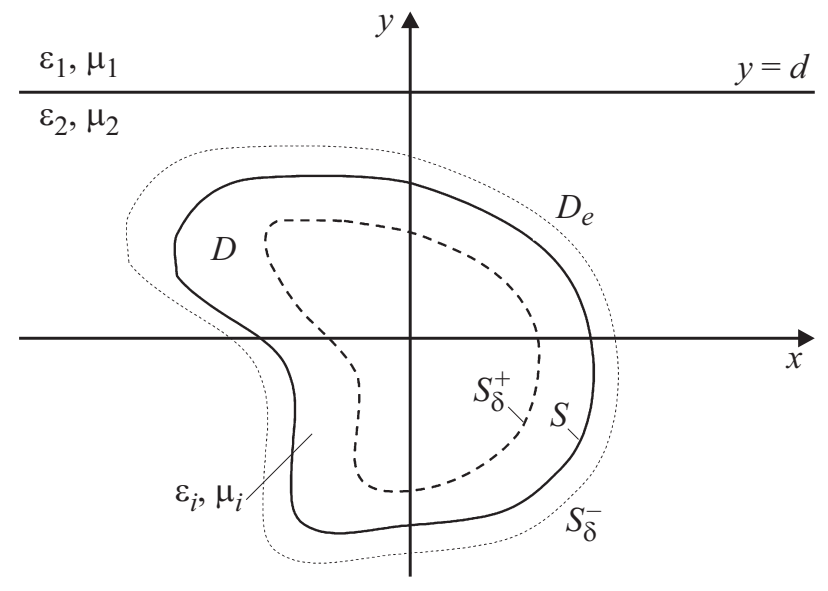

Pис. 2. Геометрия задачи дифракции на теле, расположенном в диэлектрическом полупространстве.

Формулы (13)-(17) дают два численных алгоритма (основанных на системах уравнений 1- и 2-рода) для решения сформулированной задачи дифракции.

Одним из критериев правильности полученных результатов является оптическая теорема, которая записывается в виде [11]

$$
\sigma=-\operatorname{Re}\left(g\left(\varphi=\varphi_{0}\right)\right)
$$

где

$$
\sigma=\frac{1}{2 \pi} \int_{0}^{2 \pi}|g(\varphi)|^{2} d \varphi .
$$

В качестве оценки точности выполнения оптической теоремы будем рассчитывать величину, которая представляет собой относительную разность левой и правой частей в формуле (18):

$$
\Delta_{o t} \equiv \frac{\left|\sigma+\operatorname{Re}\left(g\left(\varphi_{0}\right)\right)\right|}{\sigma} .
$$

\section{2. Рассеяние на цилиндрическом теле, погруженном в однородное диэлектрическое полупространство}

Обобщим предлагаемую методику на случай, когда рассеивающее препятствие расположено в однородном магнитодиэлектрическом полупространстве. Геометрия задачи изображена на рис. 2. Обозначим материальные параметры сред при $y>d$ и $y<d$ через $\varepsilon_{1}, \mu_{1}$ и $\varepsilon_{2}$, $\mu_{2}$ соответственно ( $y=d-$ граница раздела сред). На границе раздела предполагаются выполненными условия сопряжения

$$
\left.U\right|_{y=d}=\left.U_{-}\right|_{y=d},\left.\frac{1}{\mu_{1}} \frac{\partial U}{\partial y}\right|_{y=d}=\left.\frac{1}{\mu_{2}} \frac{\partial U_{-}}{\partial n}\right|_{y=d},
$$

где $U$ и $U_{-}-$полное поле в верхнем и нижнем полупространстве соответственно. В качестве падающего поля рассмотрим плоскую волну, падающую из верхнего полупространства $y>d$.

Как и в случае дифракции на теле, в однородной среде полное поле в нижнем полупространстве, в котором расположено тело, и поле внутри рассеивателя имеют вид (2), где функция Грина $G_{-}\left(\mathbf{r}, \mathbf{r}^{\prime}\right)$ заменяется на следующую:

$$
\begin{aligned}
G_{-}\left(\mathbf{r}, \mathbf{r}^{\prime}\right) & =-\frac{i}{4} H_{0}^{(2)}\left(k_{-}\left|\mathbf{r}-\mathbf{r}^{\prime}\right|\right)-\frac{i}{4 \pi} \int_{-\infty}^{\infty} R(w) \\
& \times \exp \left(-i \gamma_{-}\left(2 d-y-y^{\prime}\right)-i w\left(x-x^{\prime}\right)\right) \frac{d w}{\gamma_{-}} \\
& =G_{-}^{\mathrm{I}}\left(\mathbf{r} ; \mathbf{r}^{\prime}\right)+G_{-}^{\mathrm{II}}\left(\mathbf{r} ; \mathbf{r}^{\prime}\right),
\end{aligned}
$$

где $R(w)=\frac{\gamma_{-}-\mu_{21} \gamma}{\gamma_{-}+\mu_{21} \gamma}, \quad \gamma_{-}=\sqrt{k_{-}^{2}-w^{2}}, \quad \gamma=\sqrt{k^{2}-w^{2}}$. При этом знак корня выбирается так, чтобы его мнимая часть была неположительна. В приведенных формулах обозначено $k=\omega \sqrt{\varepsilon_{1} \mu_{1}}, k_{-}=\omega \sqrt{\varepsilon_{2} \mu_{2}}, \mu_{12}=\mu_{1} / \mu_{2}$, $\mu_{21}=\mu_{2} / \mu_{1}$.

Дальнейшее решение задачи вновь сводится к СИУ относительно поля и его нормальной производной на границе рассеивателя. Будем решать задачу дифракции, используя, например, СИУ 2-го рода. В результате получим СИУ в виде (8), причем ядра интегральных уравнений записываются следующим образом:

$$
\tilde{K}_{p q}=\tilde{K}_{p q}^{\mathrm{I}}+\tilde{K}_{p q}^{\mathrm{II}}, p, q=1,2,
$$

где первые слагаемые такие же, как для тела в однородной среде с волновым числом $k_{-}$, а добавочные слагаемые, обусловленные наличием границы раздела, имеют вид

$$
\begin{gathered}
\tilde{K}_{11}^{\mathrm{II}}=-\frac{1}{2} \frac{\partial G_{-}^{\mathrm{II}}\left(\mathbf{r}_{-} ; \mathbf{r}^{\prime}\right)}{\partial n^{\prime}} \xi\left(t^{\prime}\right), \tilde{K}_{12}^{\mathrm{II}}=\frac{1}{2} G_{-}^{\mathrm{II}}\left(\mathbf{r}_{-} ; \mathbf{r}^{\prime}\right) \xi\left(t^{\prime}\right), \\
\tilde{K}_{21}^{\mathrm{II}}=-\frac{1}{1+\kappa} \frac{\partial^{2} G_{-}^{\mathrm{II}}\left(\mathbf{r}_{-}, \mathbf{r}^{\prime}\right)}{\partial n \partial n^{\prime}} \xi\left(t^{\prime}\right), \\
\tilde{K}_{22}^{\mathrm{II}}=\frac{1}{1+\kappa} \frac{\partial G_{-}^{\mathrm{II}}\left(\mathbf{r}_{-}, \mathbf{r}^{\prime}\right)}{\partial n} \xi\left(t^{\prime}\right) .
\end{gathered}
$$

Кроме того, в отличие от случая дифракции на теле в однородной среде при дифракции на теле в полупространстве, первичное поле записывается следующим образом:

$$
\begin{aligned}
& U^{0}(\mathbf{r})=\frac{2 k \cos \theta_{0}}{k \cos \theta_{0}+\mu_{12} \sqrt{k_{-}^{2}-k^{2} \sin ^{2} \theta_{0}}} \\
& \times \exp \left(i k d \cos \theta_{0}-i d \sqrt{k_{-}^{2}-i^{2} \sin ^{2} \theta_{0}}\right) \\
& \times \exp \left(-i k x \sin \theta_{0}+i y \sqrt{k_{-}^{2}-k^{2} \sin ^{2} \theta_{0}}\right),
\end{aligned}
$$

где $\theta_{0}-$ угол падения плоской волны. СИУ вновь решается методом Крылова-Боголюбова, однако в силу 
того, что добавочные ядра интегральных уравнений являются медленно меняющимися функциями координат, матричные элементы СЛАУ можно вычислять по приближенной формуле

$$
\tilde{K}_{m n}^{p q} \approx \int_{t_{n}-\Delta / 2}^{t_{n}+\Delta / 2} \tilde{K}_{p q}^{\mathrm{I}}\left(t_{m}, t\right) d t+\tilde{K}_{p q}^{\mathrm{II}}\left(t_{m}, t_{n}\right) \Delta, p, q=1,2 .
$$

Приведем формулы для расчета диаграммы рассеяния в верхнем полупространстве. Диаграмма имеет вид

$$
\begin{aligned}
g(\varphi) & =\frac{i \Delta}{4} \frac{2 k \sin \varphi}{\mu_{21} k \sin \varphi+\sqrt{k_{-}^{2}-k^{2} \cos ^{2} \varphi}} \\
& \times \exp \left(i k d \sin \varphi-i d \sqrt{\left.k_{-}^{2}-k^{2} \cos ^{2} \varphi\right)}\right. \\
& \times \sum_{n=1}^{N}\left(c_{n}^{2}-c_{n}^{1}\left(i n_{x}^{\prime}\left(t_{n}\right) k \cos \varphi\right.\right. \\
& \left.\left.+i n_{y}^{\prime}\left(t_{n}\right) \sqrt{k_{-}^{2}-k^{2} \cos ^{2} \varphi}\right)\right) \\
& \left.\times e^{i\left(k x^{\prime}\left(t_{n}\right) \cos \varphi+y^{\prime}\left(t_{n}\right) \sqrt{k_{-}^{2}-k^{2} \cos ^{2} \varphi}\right.}\right)_{\xi\left(t_{n}\right) .}
\end{aligned}
$$

\section{3. Численные результаты}

Рассмотрим результаты численного моделирования. Всюду в дальнейшем будем предполагать, что тело облучается плоской волной. В качестве примера рассмотрим сначала задачу дифракции на эллиптическом цилиндре, цилиндре с сечением в виде четырехлистника и цилиндре с прямоугольным сечением. Уравнение контура тела с сечением в виде четырехлистника имеет вид (в полярных координатах)

$$
r=a(1+\tau \cos 4 \varphi), 0<\tau<1 .
$$

На рис. 3-5 приведены угловые зависимости диаграммы рассеяния для соответствующей геометрии, полученные для следующих значений параметров задачи: $k \delta=10^{-4}, \varphi_{0}=0, \mu_{i}=1, \varepsilon_{i}=4$ (материальные параметры внешней среды всюду $\left.\mu_{e}=1, \varepsilon_{e}=1\right)$. Размеры тел имели следующие значения: полуоси эллипса или половины длин сторон прямоугольника $k a=5, k b=1$, параметры $k a=5$ и $\tau=0.5$ для тела с сечением в виде четырехлистника. Результаты сравнивались с диаграммами, построенными при помощи модифицированного метода дискретных источников (ММДИ) [5,12]. Отметим, что ММДИ не может быть непосредственно применен к задаче дифракции на телах, имеющих изломы границы, поэтому для решения задачи при помощи ММДИ проводилась аппроксимация контура осевого сечения тела гладким контуром [12]. Отметим также, что ММДИ обеспечивает высокую точность расчета для тел с гладкой границей таких, как эллипс, многолистник и т.д.

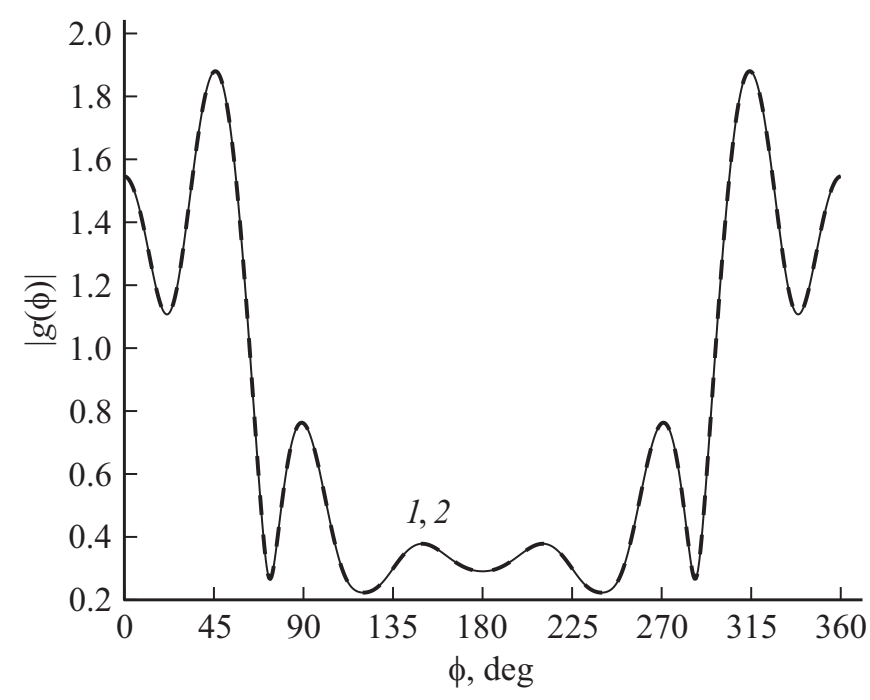

Рис. 3. Угловая зависимость диаграммы рассеяния эллиптического цилиндра. Кривая 1 - ММДИ, кривая 2 - МПГУ.

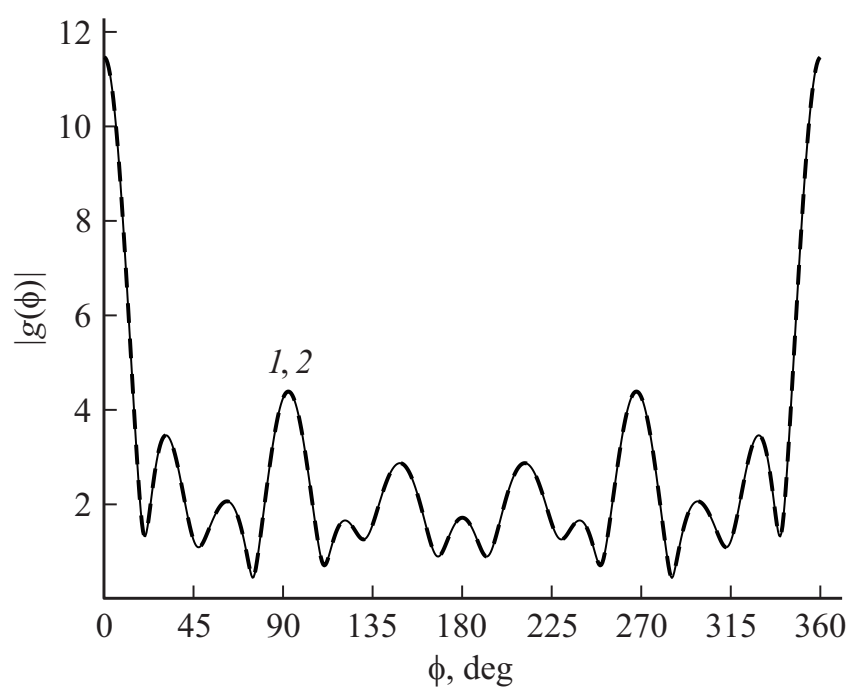

Рис. 4. Угловая зависимость диаграммы рассеяния тела с сечением в виде четырехлистника. Кривая $1-$ ММДИ, кривая $2-$ МПГУ.

Таблица 1. Сравнение результатов, полученных при помощи ММДИ и МПГУ. Дифракция на эллиптическом цилиндре

\begin{tabular}{c|c|c|c|c}
\hline \multirow{2}{*}{$\mathrm{N}$} & \multicolumn{2}{|c|}{ СИУ 1-го рода } & \multicolumn{2}{c}{ СИУ 2-го рода } \\
\cline { 2 - 5 } & $\begin{array}{c}\text { Абсолютная } \\
\text { погрешность }\end{array}$ & $\begin{array}{c}\text { Относительная } \\
\text { погрешность }\end{array}$ & $\begin{array}{c}\text { Абсолютная } \\
\text { погрешность }\end{array}$ & $\begin{array}{c}\text { Относительная } \\
\text { погрешность }\end{array}$ \\
\hline 48 & $1.295 \cdot 10^{-2}$ & $2.038 \%$ & $1.453 \cdot 10^{-1}$ & $24.297 \%$ \\
96 & $1.904 \cdot 10^{-3}$ & $0.230 \%$ & $4.183 \cdot 10^{-2}$ & $7.238 \%$ \\
192 & $6.096 \cdot 10^{-4}$ & $0.067 \%$ & $1.144 \cdot 10^{-2}$ & $2.003 \%$ \\
288 & $5.834 \cdot 10^{-4}$ & $0.075 \%$ & $5.539 \cdot 10^{-3}$ & $0.977 \%$ \\
384 & $5.607 \cdot 10^{-4}$ & $0.075 \%$ & $3.450 \cdot 10^{-3}$ & $0.612 \%$
\end{tabular}




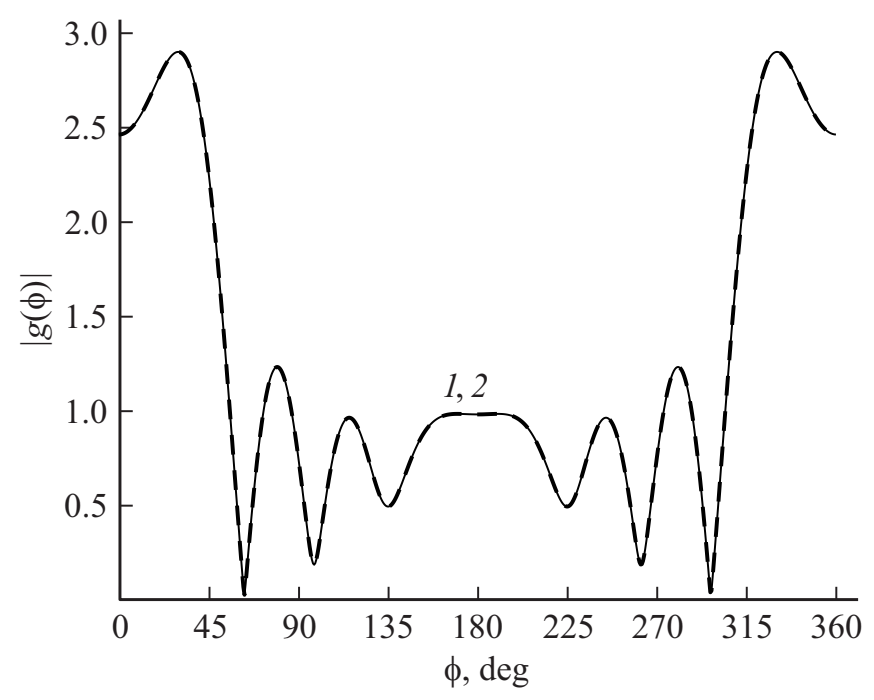

Рис. 5. Угловая зависимость диаграммы рассеяния тела с прямоугольным сечением. Кривая 1 - ММДИ, кривая $2-$ МПГУ.

Таблица 2. Сравнение результатов, полученных при помощи ММДИ и МПГУ. Дифракция на теле с сечением в виде четырехлистника

\begin{tabular}{c|c|c|c|c}
\hline \multirow{2}{*}{$\mathrm{N}$} & \multicolumn{2}{|c|}{ СИУ 1-го рода } & \multicolumn{2}{c}{ СИУ 2-го рода } \\
\cline { 2 - 5 } & $\begin{array}{c}\text { Абсолютная } \\
\text { погрешность }\end{array}$ & $\begin{array}{c}\text { Относительная } \\
\text { погрешность }\end{array}$ & $\begin{array}{c}\text { Абсолютная } \\
\text { погрешность }\end{array}$ & $\begin{array}{c}\text { Относительная } \\
\text { погрешность }\end{array}$ \\
\hline 48 & $1.643 \cdot 10^{-1}$ & $10.411 \%$ & $3.836 \cdot 10^{-1}$ & $21.169 \%$ \\
96 & $2.499 \cdot 10^{-2}$ & $1.442 \%$ & $9.462 \cdot 10^{-2}$ & $5.125 \%$ \\
192 & $5.802 \cdot 10^{-3}$ & $0.325 \%$ & $2.534 \cdot 10^{-2}$ & $1.370 \%$ \\
288 & $2.984 \cdot 10^{-3}$ & $0.166 \%$ & $1.143 \cdot 10^{-2}$ & $0.619 \%$ \\
384 & $2.176 \cdot 10^{-3}$ & $0.121 \%$ & $6.474 \cdot 10^{-3}$ & $0.351 \%$
\end{tabular}

Таблица 3. Сравнение результатов, полученных при помощи ММДИ и МПГУ. Дифракция на теле с прямоугольным сечением

\begin{tabular}{c|c|c|c|c}
\hline \multirow{2}{*}{$\mathrm{N}$} & \multicolumn{2}{|c|}{ СИУ 1-го рода } & \multicolumn{2}{c}{ СИУ 2-го рода } \\
\cline { 2 - 5 } & $\begin{array}{c}\text { Абсолютная } \\
\text { погрешность }\end{array}$ & $\begin{array}{c}\text { Относительная } \\
\text { погрешность }\end{array}$ & $\begin{array}{c}\text { Абсолютная } \\
\text { погрешность }\end{array}$ & $\begin{array}{c}\text { Относительная } \\
\text { погрешность }\end{array}$ \\
\hline 48 & $3.498 \cdot 10^{-2}$ & $4.781 \%$ & $5.035 \cdot 10^{2}$ & $6.795 \%$ \\
96 & $1.466 \cdot 10^{-2}$ & $1.956 \%$ & $1.417 \cdot 10^{-2}$ & $1.715 \%$ \\
192 & $7.358 \cdot 10^{-3}$ & $0.879 \%$ & $4.773 \cdot 10^{-3}$ & $0.466 \%$ \\
288 & $5.229 \cdot 10^{-3}$ & $0.561 \%$ & $3.122 \cdot 10^{-3}$ & $0.248 \%$ \\
384 & $4.219 \cdot 10^{-3}$ & $0.429 \%$ & $2.641 \cdot 10^{-3}$ & $0.208 \%$
\end{tabular}

В табл. 1-3 приведены разности модуля диаграммы рассеяния для указанной геометрии, полученные двумя методами: при помощи ММДИ и МПГУ. Как видно из табл. 1-3, разность результатов при увеличении числа используемых базисных функций, уменьшается. Из приведенных данных также следует, что для тел с гладкой границей использование уравнений 1-го рода является более предпочтительным в силу более быстрой сходимости. В случае тела с прямоугольным сечением использование уравнений 2-го рода дает лучшие результаты.

На рис. 6 приведена геометрия фракталоподобных цилиндров с сечением в виде снежинки Коха и кривой Серпинского (1 итерация) [13]. Рисунки 7 и 8 иллюстрируют угловые зависимости диаграммы рассеяния для указанных цилиндров для следующих параметров задачи $k \delta=10^{-4}, \mu_{i}=1, \varepsilon_{i}=4$. Максимальный поперечный размер тела с сечением в виде снежинки Коха и тела с сечением в виде кривой Серпинского по оси $x$ составлял $k L=10$. Рассматривалось два различных угла падения: $\varphi_{0}=0$ и $\varphi_{0}=45^{\circ}$. Как следует из рисунков для исследуемой геометрии точки максимума угловых зависимостей диаграммы рассеяния примерно совпадают с углами падения плоской волны. Видно также, что зависимость диаграммы как для тела с сечением в виде снежинки Коха, так и для тела с сечением в виде

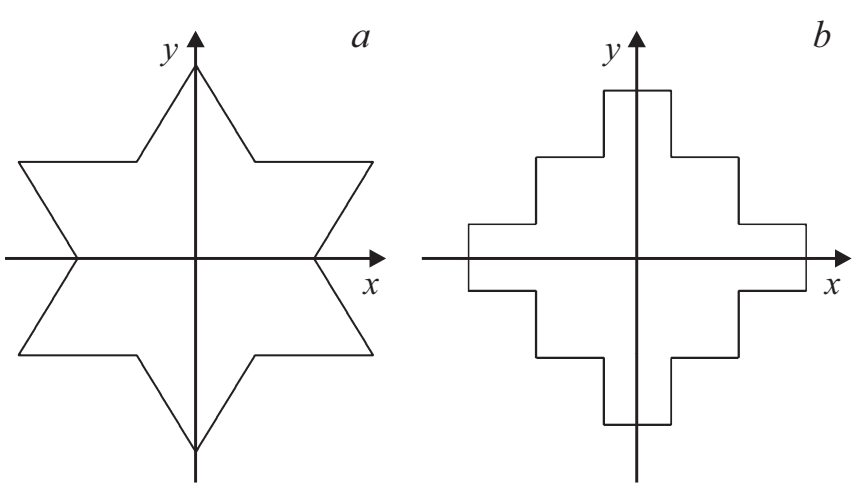

Рис. 6. Геометрия тела с сечением в виде снежинки Коха $(a)$ и кривой Серпинского $(b)$.

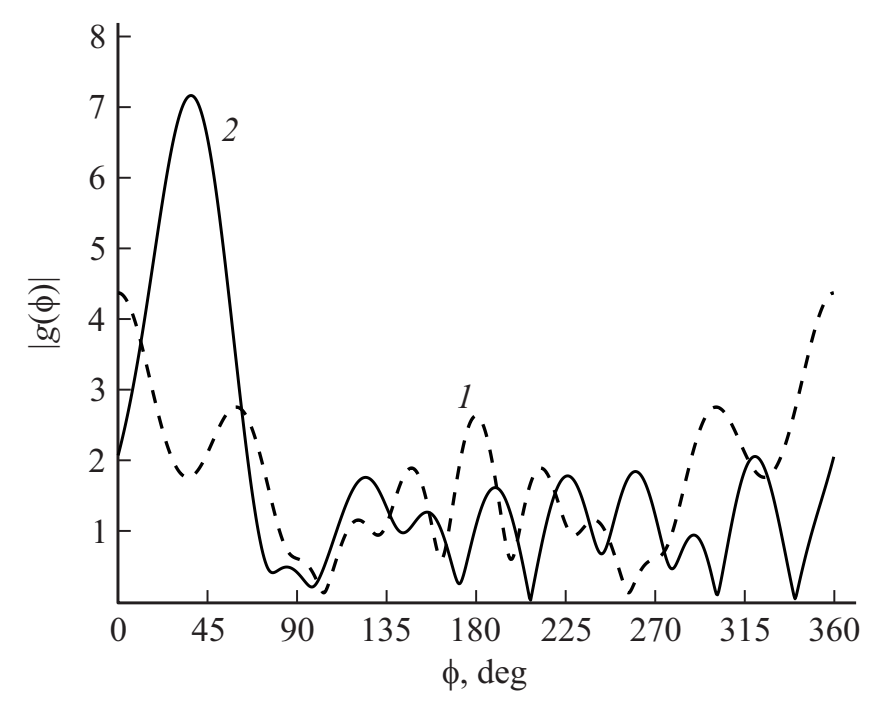

Рис. 7. Угловая зависимость диаграммы рассеяния тела с сечением в виде снежинки Коха. Кривая $1-$ угол падения волны $\varphi_{0}=0^{\circ}$, кривая $2-$ угол падения $\varphi_{0}=45^{\circ}$. 
Таблица 4. Сравнение результатов, полученных при помощи ММДИ и МПГУ. Дифракция на теле, расположенном в диэлектрическом полупространстве

\begin{tabular}{c|c|c|c|c|c|c}
\hline \multirow{2}{*}{$\mathrm{N}$} & \multicolumn{2}{|c|}{ Эллиптический цилиндр } & \multicolumn{2}{|c|}{$\begin{array}{c}\text { Цилиндр с сечением } \\
\text { четырехлистника }\end{array}$} & \multicolumn{2}{|c}{$\begin{array}{c}\text { Цилиндр с прямоугольным } \\
\text { сечением }\end{array}$} \\
\cline { 2 - 7 } & $\begin{array}{c}\text { Абсолютная } \\
\text { погрешность }\end{array}$ & $\begin{array}{c}\text { Относительная } \\
\text { погрешность }\end{array}$ & $\begin{array}{c}\text { Абсолютная } \\
\text { погрешность }\end{array}$ & $\begin{array}{c}\text { Относительная } \\
\text { погрешность }\end{array}$ & $\begin{array}{c}\text { Абсолютная } \\
\text { погрешность }\end{array}$ & $\begin{array}{c}\text { Относительная } \\
\text { погрешность }\end{array}$ \\
\hline 48 & $3.616 \cdot 10^{-2}$ & $9.047 \%$ & $3.414 \cdot 10-^{1}$ & $51.927 \%$ & $2.146 \cdot 10^{-2}$ & $6.856 \%$ \\
96 & $1.057 \cdot 10^{-2}$ & $2.622 \%$ & $1.235 \cdot 10^{-1}$ & $18.859 \%$ & $4.928 \cdot 10^{-3}$ & $1.611 \%$ \\
192 & $3.073 \cdot 10^{-3}$ & $0.735 \%$ & $3.609 \cdot 10^{-2}$ & $5.696 \%$ & $2.432 \cdot 10^{-3}$ & $0.359 \%$ \\
288 & $1.616 \cdot 10^{-3}$ & $0.368 \%$ & $1.687 \cdot 10^{-2}$ & $2.670 \%$ & $2.121 \cdot 10^{-3}$ & $0.354 \%$ \\
384 & $1.099 \cdot 10^{-3}$ & $0.238 \%$ & $9.829 \cdot 10^{-3}$ & $1.548 \%$ & $2.630 \cdot 10^{-3}$ & $0.377 \%$
\end{tabular}

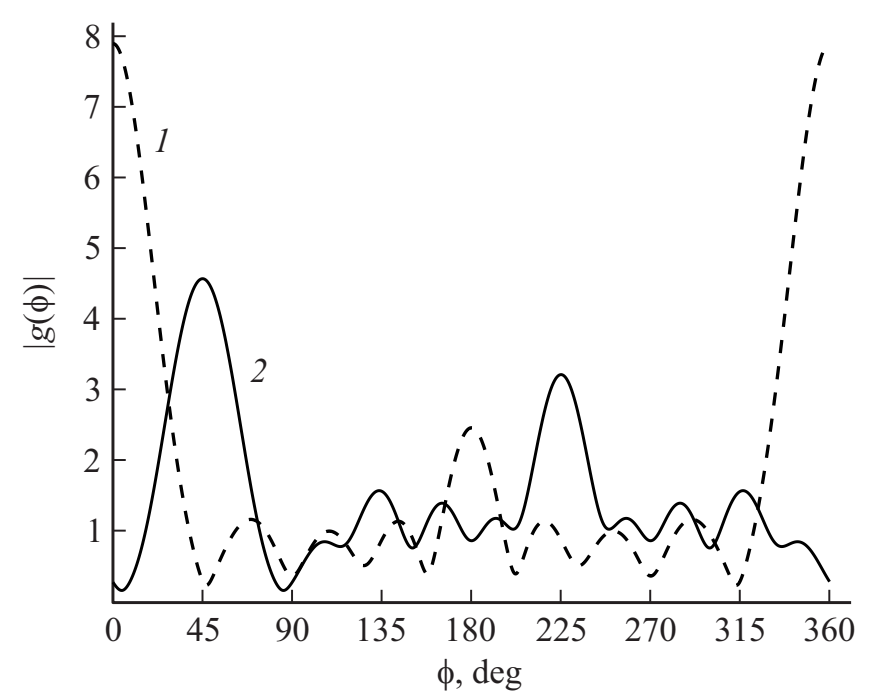

Рис. 8. Угловая зависимость диаграммы рассеяния тела с сечением в виде кривой Серпинского. Кривая 1 - угол падения волны $\varphi_{0}=0^{\circ}$, кривая $2-$ угол падения $\varphi_{0}=45^{\circ}$.

кривой Серпинского имеет достаточно большие боковые лепестки.

Была проведена проверка точности выполнения оптической теоремы для рассмотренной выше геометрии рассеивателей. Во всех случаях мы выбирали число базисных функций так, что $N_{\lambda}=25$, где $N_{\lambda}$ - число точек коллокации на одной длине волны. При этом относительная диэлектрическая проницаемость среды тела варьировалась в пределах от $\varepsilon_{i}=4$ до $\varepsilon_{i}=10^{3}$, а относительная магнитная проницаемость выбиралась равной единице. В результате расчетов получили, что относительная разность правой и левой частей равенства (18) - величина $\Delta_{o t}$ (см. формулу (20)) - не превосходит $5 \cdot 10^{-3}$, т. е. мала.

В табл. 4 приведены результаты расчета диаграммы рассеяния, полученные при помощи МПГУ и ММДИ. Рассматривалась дифракция на теле, расположенном в диэлектрическом полупространстве. Размеры тел были выбраны такими же, как и в случае дифракции в
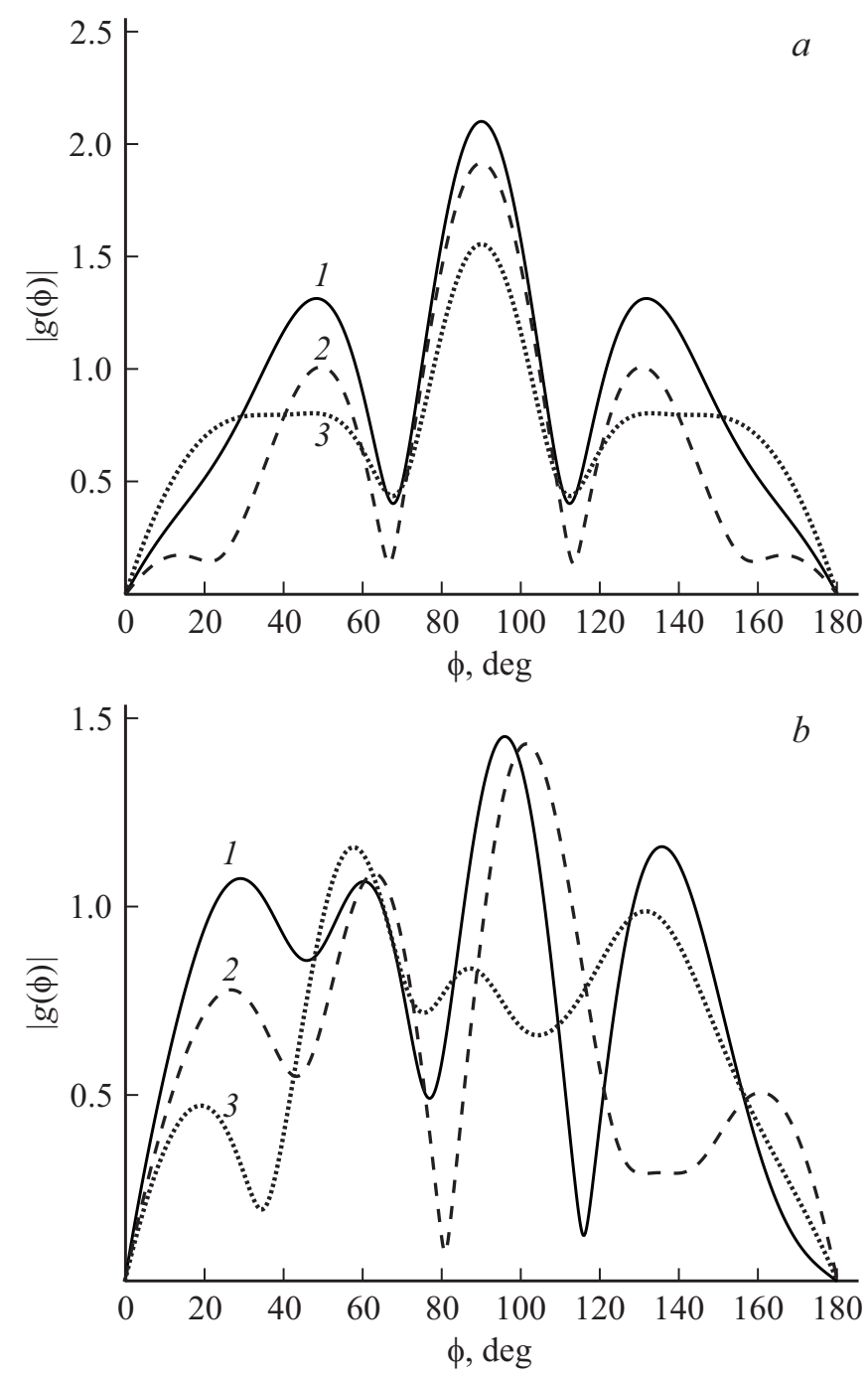

Рис. 9. Угловая зависимость диаграммы рассеяния тела, расположенного в диэлектрическом полупространстве. Кривая 1 - цилиндр с сечением в виде кривой Серпинского, кривая 2 - цилиндр с сечением в виде снежинки Коха, кривая 3 - цилиндр с сечением в виде правильного шестиугольника. Угол падения $\theta_{0}=0(a)$ и $45^{\circ}(b)$. 
однородной среде, а материальные параметры сред для верхнего и нижнего полупространств и цилиндрического тела имели следующие значения: $\mu_{1}=1, \varepsilon_{1}=1, \mu_{2}=1$, $\varepsilon_{2}=2-i \cdot 10^{-3}, \mu_{i}=1, \varepsilon_{i}=6$. Величина $d$ была выбрана так, что кратчайшее расстояние от границы всех тел до границы раздела сред составляло 1. Параметр $k \delta=10^{-4}$. Из таблицы следует, что относительная разность результатов, полученных при помощи МПГУ и ММДИ, не превосходит 1.6\%. На рис. 9 приведены угловые зависимости диаграммы рассеяния для цилиндров с сечением в виде правильного шестиугольника, снежинки Коха и кривой Серпинского (1 итерация), расположенных в однородном полупространстве. Зависимости диаграммы приведены для верхнего полупространства. Рассматривалось два различных угла падения плоской волны: $\theta_{0}=0$ и $\theta_{0}=45^{\circ}$. Как следует из рисунка, в случае нормального падения плоской волны диаграмма рассеяния для всех тел имеет главный лепесток (в направлении обратного рассеяния) и два боковых лепестка. В случае наклонного падения волны график диаграммы имеет осциллирующий характер.

\section{Заключение}

При помощи МПГУ разработаны два численных алгоритма на основе СИУ 1-го и 2-го рода, позволяющие рассчитывать характеристики рассеяния магнитодиэлектрических тел произвольной геометрии. Получены результаты расчета диаграммы рассеяния для большого набора тел с разной геометрией, в том числе фракталоподобных рассеивателей. Проведено сравнение методов на основе МПГУ с результатами, полученными при помощи ММДИ. МПГУ позволяет получать результаты расчета диаграммы рассеяния с достаточно высокой точностью. В случае гладкой границы тела метод на основе уравнений 1-го рода позволяет получать результаты с большей точностью. Проведена проверка точности выполнения оптической теоремы для рассматриваемой геометрии. Точность выполнения оптической теоремы составляет $5 \cdot 10^{-3}$. Сравнение ММДИ и МПГУ для случая дифракции на цилиндрическом теле, расположенном в диэлектрическом полупространстве, показало хорошее совпадение результатов расчета. Построены угловые зависимости диаграммы рассеяния для тел, имеющих изломы границы, расположенных в диэлектрическом полупространстве.

\section{Финансирование работы}

Работа выполнена при частичной поддержке Российского фонда фундаментальных исследований (проекты № 18-02-00961, 19-02-00654).

\section{Конфликт интересов}

Авторы заявляют, что у них нет конфликта интересов.

\section{Список литературы}

[1] Борен К., Хаффмен Д. Поглощение и рассеяние света малыми частицами. М.: Мир, 1986.; Bohren K.F., Huffman D.R. Absorption and Scattering of Light by Small Particles. N.Y.: John Wiley \& Sons, 1983.

[2] Захарьев Л.Н., Леманский А.А. Рассеяние волн „черными“ телами. М.: Сов. радио, 1972.

[3] Mishchenko M.I., Zakharova N.T., Khlebtsov N.G., Videen G., Wriedt T. // J. Quant. Spectr. Rad. Trans. 2017. V. 202. P. 240.

[4] Waterman P.C. // Proc. IEEE. 1965. V. 53. P. 805.

[5] Кюркчан А.Г., Смирнова Н.И. Математическое моделирование в теории дифракции с использованием априорной информации об аналитических свойствах решения. М.: ИД Медиа Паблишер, 2014; Kyurkchan A.G., Smirnova N.I. Mathematical Modeling in Diffraction Theory Based on A Priori Information on the Analytical Properties of the Solution. Amsterdam: Elsevier, 2016

[6] Кюркчан А.Г., Смирнова Н.И., Чиркова А.П. // РЭ. 2015. T. 60. № 3. C. 247; Kyurkchan A.G., Smirnova N.I., Chirkova A.P. // J. Commun. Technol. Electron. 2015. V. 60. N 3. P. 232.

[7] Кюркчан А.Г., Смирнова Н.И. // РЭ. 2017. Т. 62. № 5. C. 476; Kyurkchan A.G., Smirnova N.I. // J. Commun. Technol. Electron. 2017. V. 62. N 5. P. 502.

[8] Кюркчан А.Г., Маненков С.А., Смирнова Н.И. // Опт. и спектр. 2019. Т. 126. № 5. С. 547; Kyurkchan A.G., Manenkov S.A., Smirnova N.I. // Opt. and Spectrosc. 2019. V. 126. N 5. P. 466.

[9] Крысанов Д.В., Кюркчан А.Г. // Т-Сотm. Телекоммуникации и транспорт. 2017. Т. 11. № 7. С. 17.

[10] Кюркчан А.Г., Анютин А.П. // ДАН. 2002. Т. 385. № 3. C. 309; Kyurkchan A.G., Anyutin A.P. // Doklady Mathematics. 2002. V. 66. N 1. P. 132.

[11] Шендеров Е.Л. Излучение и рассеяние звука. Л.: Судостроение, 1989.

[12] Kyurkchan A.G, Manenkov S.A. // J. Quant. Spectrosc. Radiat. Transfer. 2012. V. 113. P. 2368.

[13] Кроновер Р.М. Фракталы и хаос в динамических системах. Основы теории. М.: Постмаркет, 2000; Crownover R.M. Intoduction to Fractals and Chaos. Boston: Jones and Bartlett Publishers, 1995. 